Voix et Images

voixetimages

\title{
Entretien avec André Major
}

\section{Élisabeth Nardout-Lafarge}

Volume 40, numéro 3 (120), printemps-été 2015

André Major

URI : https://id.erudit.org/iderudit/1032631ar

DOI : https://doi.org/10.7202/1032631ar

Aller au sommaire du numéro

\section{Éditeur(s)}

Université du Québec à Montréal

\section{ISSN}

0318-9201 (imprimé)

1705-933X (numérique)

Découvrir la revue

\section{Citer ce document}

Nardout-Lafarge, É. (2015). Entretien avec André Major. Voix et Images, 40(3), 13-27. https://doi.org/10.7202/1032631ar d'utilisation que vous pouvez consulter en ligne.

https://apropos.erudit.org/fr/usagers/politique-dutilisation/ 


\title{
ENTRETIEN AVEC ANDRÉ MAJOR
}

\author{
$+++$ \\ ÉLISABETH NARDOUT-LAFARGE \\ Université de Montréal
}

VoIX ET IMAGES Votre œuvre est en quelque sorte tendue entre le choix de la fiction (romans, nouvelles) et son refus (les carnets). Or si l'écriture des carnets est explicitement donnée comme «l'adieu au roman», vous y êtes revenu avec À quoi ça rime? Est-ce à dire que le romanesque et le fictif ne peuvent pas être totalement abandonnés?

ANDRÉ MAJOR Il y a toujours eu chez moi une tension entre la fiction et la prose journalière, si je puis me permettre ce qualificatif, même à l'époque de mes premières tentatives, qui remontent à mon adolescence. D'une part, je voyais dans la fiction un espace de liberté quasi illimité et, d'autre part, dans le journal ou le carnet, je m'inspirais de l'expérience vécue, entendue au sens très large et incluant aussi bien l'observation que la lecture et la réflexion. Mais dans les deux cas, ce qui a toujours été l'enjeu de l'écriture, c'est l'interprétation d'un réel et d'un moi aussi ténébreux l'un que l'autre. Bien qu'écrire avec le souci d'y voir plus clair finisse par ouvrir certaines brèches, aménager une éclaircie ici et là, grâce à la liberté et à l'espèce d'immunité qu'octroie le recours à l'imaginaire, et que la prose des carnets, de son côté, permette de mesurer le terrain gagné peu à peu sur les ténèbres, je ne considère pas l'écriture comme la voie d'un quelconque salut. Mais cette tension entre la fiction et la prose émiettée des carnets - qui coexiste d'ailleurs avec une tension entre la campagne et la ville - me semble plus féconde que problématique, parce que c'est dans cette double tension que je trouve un certain équilibre. S'il n'y avait pas eu ce qu'Ernst Jünger appelle «le recours aux forêts» dans mon enfance, puis tout au long de ma vie, je n'aurais pas développé le même imaginaire. Encore aujourd'hui, c'est en arpentant la montagne par des sentiers inusités qu'une histoire me vient. Une fois ensemencée, peu m'importe le lieu de sa gestation. Quand j'ai décidé de renoncer au roman, c'était pour jouir pleinement de la retraite, en ne me livrant plus à l'écriture que pour préserver de l'oubli ceci ou cela. Si je ne voulais plus m'astreindre à la discipline qu'exige le travail romanesque, je n'en demeurais pas moins aux aguets, si je puis dire, de tout ce qui pouvait se traduire en observations éparses que je traitais avec la même rigueur esthétique que s'il s'agissait d'un roman ou d'une nouvelle. Car les carnets ne peuvent être un simple recueil de miettes à peine toilettées et présentées selon un ordre chronologique. Il me faut procéder à un premier tri dans ce 
fourre-tout, avant d'en retenir ce qui me semble mériter une réécriture aussi rigoureuse que possible, jusqu'à ce que le résultat réponde aux exigences de cette prose évocatrice et précise qu'est en droit d'attendre le lecteur qui chemine à mon côté. Car écrire sur le mode du monologue, ce ne peut être pour soi seul, même si l'élan initial nous porte à nous adresser à nous-même.

voIX ET IMAGES Dans la série d'entretiens avec Stéphane Lépine diffusée à la Chaîne culturelle de Radio-Canada ${ }^{1}$, évoquant la différence entre "écriture intimiste» et «écriture de l'intime», vous dites que le «il» de la fiction instaure la distance qui permet au moi de devenir autre alors que le «je» engage dans l'intime. Le choix du «je» pourtant fictif d'À quoi ça rime? vient-il redéfinir ou nuancer cette distinction? ANDRÉ MAJOR Le point de vue narratif est une question cruciale pour tout écrivain, si son mode narratif n'est pas déterminé une fois pour toutes. Dans mes histoires longues ou courtes, la narration se veut objective, le narrateur demeurant invisible, comme s'il se contentait de braquer la caméra sur l'action. Sauf peut-être dans Le cabochon, mon premier roman publié, où, comme Gérard Bessette le prétendait, le narrateur est un «je» masqué à travers le regard duquel tout se trouve filtré. Dans certaines nouvelles de La chair de poule, un narrateur intempestif interpelle le lecteur pour le provoquer et lui rappeler tout ce qui le distingue des personnages sur lesquels il le convie à s'apitoyer. Ces œuvres, marquées au fer rouge d'un certain militantisme, je n'ai pas d'autre choix que d'en assumer la paternité. Au cours des années qui suivront, je chercherai une autre voie, en même temps qu'une voix plus neutre, bien que Le vent du diable, par exemple, soit assez lyrique, du moins dans la première partie racontée à la troisième personne. Paradoxalement, c'est dans l'épilogue intitulé «Le cahier bleu", où le protagoniste se démasque en avouant les sentiments qu'il éprouve à l'égard de la femme à qui il s'adresse, que le ton devient résolument réaliste. Je considère cet opus comme un ouvrage de transition, qui porte les traces assez visibles de mes lectures de cette période (Savard, Hesse, Giono et Ramuz). D'autres lectures me pousseront à aborder le récit autrement en travaillant sur la composition et le dialogue (Flaubert, Faulkner, les grands prosateurs russes et les maîtres du roman noir). Durant un séjour d'un an à Toulouse, je tâtonne beaucoup, passant d'un récit autobiographique avorté à un roman, Le coureur de nuit, configuré pour la Série noire de Gallimard. Mais je ne livre pas la marchandise, après avoir compris que cette histoire appelle un traitement plus sophistiqué, qui m'engage plus profondément dans la forme romanesque dont je rêve. Finalement, je passe un mois sous la tente, non loin du chalet de mes parents, à remanier de fond en comble ce récit porté par une sorte d'allégresse féconde, comme je l'ai déjà écrit quelque part. Ce sera L'épouvantail, le premier volet de mes Histoires de déserteurs dont je poursuis la rédaction jusqu'en 1976. Mais mon élan retombe parce que je suis très sollicité par les responsabilités familiales, par mon travail de réalisateur, que je mène tout en poursuivant mon travail de chroniqueur littéraire, en dirigeant des ateliers d'écriture et en exécutant du

1 «Les Carnets d'André Major. Une série en 8 volets», La 11e heure, émissions animées par Stêphane Lépine, Société Radio-Canada, du 15 au 19, puis du 22 au 24 décembre 1997, nos 1260085, 1260305, 1260306, 1259942, 1259944, 1798666, 1798664 et 1798661 du centre d'archives Gaston-Miron, http://www.crlq. umontreal.ca (fiches consultées le 22 avril 2015). 
travail d'édition à l'occasion. Une pause s'impose donc, mais le souci de l'écriture ne fait pas relâche pour autant. Je lis beaucoup de nouvellistes dont les approches me ramènent à la nouvelle. J'en écris quelques-unes pour des revues, j'en écris d'autres que je remanie, jusqu'à ce qu'une relecture me laisse entrevoir un fil conducteur assurant la cohérence d'un ensemble. J'en retiens une douzaine qui paraissent sous le titre de La folle d'Elvis. Une seule est écrite à la première personne par un narrateur qui raconte un rêve et l'effet qu'il a eu sur lui. Cette question du point de vue narratif m'a longtemps préoccupé, mais j'en suis venu à la conclusion que, dans tous les cas, l'écrivain est un monologuiste, qui tente, avec les moyens les plus appropriés à son propos, d'amener le lecteur dans son univers, de le tenir sous le charme de sa parole. Toute son ambition est d'être enfin écouté, d'enchanter son lecteur, de le faire rêver comme devant un tableau. Pour en venir enfin au «je» d'Ā quoi ça rime?, on pourrait supposer que ce roman inattendu a été contaminé par le ton intimiste de Prendre le large, que je venais de terminer. Au départ, c'est une nouvelle que je voulais écrire, le bref récit d'un deuil raconté par Antoine, mon alter ego du Cabochon et de L'hiver au cœur. Le fil de l'histoire s'est déroulé de manière tout à fait imprévisible, et force m'a été de constater que j'étais en train d'écrire un roman. J'ai alors passé le relais à la troisième personne, jusqu'à ce que je prenne conscience que ce changement me coupait littéralement le souffle et que le ton changeait. Ce récit, hanté par le passé et par l'avenir, exigeait un phrasé ample que ruinait le recours au «il». Toujours est-il que, pour retrouver mon élan narratif initial, je suis revenu à ce «je» que certaines lectures déterminantes (Thomas Bernhard et W. G. Sebald) avaient réhabilité à mes yeux. Si je donne une suite à À quoi ça rime? ce sera probablement en recourant au même mode narratif et à un imaginaire nourri autant de ma propre expérience que de mon observation. C'est dans ces conditions-là que je crois être au meilleur de ma forme.

voIX ET IMAGES Vous avez pratiqué à la fois des formes brèves (la nouvelle) et des formes plus amples (le roman, mais aussi le cycle, la trilogie). Comment se distribuent ces formes et en quoi répondent-elles à des exigences différentes?

ANDRÉ MAJOR D'une manière générale, le roman s'impose quand la situation que j'entends développer met en scène plusieurs protagonistes et, surtout, quand cette situation se déploie sur une assez longue période. S'il s'agit d'une trame centrée sur un personnage et que celui-ci traverse une crise de courte durée, c'est la forme brève qui convient. Il y a des histoires qui s'inscrivent entre ces deux formats, comme c'est le cas de L'hiver au cœur, épisode tiré d'un roman inachevé parce qu'il me conduisait dans une sorte de no man's land. Quand un personnage ne me lâche plus, il me suffit d'examiner le lieu où il évolue, ce qui le meut et ce qu'il tente de me dire, pour savoir à quoi m'en tenir en ce qui concerne la durée que je lui réserve. Il est vrai qu'en écrivant À quoi ça rime? 'j'ai amené Antoine à dessiner les plans de la cabane qui lui servirait d'ermitage à son retour au pays, ce qui me contraignait à poursuivre ce récit qui ne pouvait plus s'achever comme prévu avec la fin de son séjour à Lisbonne. Tchékhov disait, si je me souviens bien, que s'il y a un fusil sur la scène, un coup de feu doit éclater à un moment donné.

VoIX ET IMAGES La critique a souvent parlé de retenue, de dépouillement, de sobriété, de mode "mineur» à propos de votre style. Votre phrase classique, tenue, qui se 
refuse aux effets, signe votre singularité, surtout dans le contexte esthétique de l'écriture «flamboyante» des années 1960-1970 au Québec. Comment travaillezvous sur ce plan?

ANDRÉ MAJOR C'est en relisant certains de mes livres, à l'occasion de leur réédition, que j'ai peu à peu pris conscience de mes forces et de mes faiblesses stylistiques. Cette conscience des mots, je l'ai développée également au cours de mes lectures, et la plus importante, je dirais que c'est celle de Tchékhov, même si je ne le lis pas dans sa langue. Son influence a été déterminante sur le plan esthétique autant que sur le plan éthique. Pour résister comme il l'a fait aux séductions idéologiques qui aveuglaient la classe intellectuelle en s'en tenant à une observation lucide et sans complaisance de la société russe, il fallait être doué d'une grande force de caractère et surtout d'une conscience aiguë du rôle de l'artiste. Quand je sombre dans la morosité - ce qui m'arrive de loin en loin -, je relis sa correspondance et je ne tarde pas à refaire surface. C'est grâce à lui, à son souci de dire les choses telles qu'il les voyait, sans surenchère verbale, mais avec cette ironie très fine, parfois dévastatrice, qui choquait tant les prédicateurs à la Chestov, que je me suis cantonné dans un rôle d'observateur - mais, bien entendu, l'observateur n'échappe pas à l'interprétation. La seule faiblesse de ce cher Anton consistait à croire que le progrès scientifique finirait par avoir raison de tout obscurantisme. Mais, pour en revenir à l'écriture flamboyante dont vous parlez, elle n'aura été qu'un feu de paille, qui ne m'a guère impressionné. Je me suis toujours ennuyé en lisant les écrivains empêtrés dans les maniérismes à la mode. Ces prétendus virtuoses ont d'ailleurs filé comme des comètes, bientôt éclipsés par d'autres funambules si peu ancrés dans le réel qu'il leur fallait forger des mots qui en tenaient lieu. À égale distance des minimalistes et des adeptes de la boursoufflure, je suis toujours à la recherche d'une respiration réglée sur le mode narratif que j'adopte et qui varie, comme on peut le constater dans le phrasé ample et plus complexe qu'il n'y paraît des Histoires de déserteurs, comparé à celui de la plupart de mes nouvelles. Comme romancier, j'ai tendance à privilégier une construction de type faulknérien, d'où ma prédilection pour les œuvres de Juan Carlos Onetti, de Juan José Saer, de Thomas Bernhard, de Peter Handke, et, plus récemment, de W. G. Sebald. Si je puis me vanter d'une chose, c'est d'avoir assez tôt veillé à ce que ma langue plonge le lecteur dans l'épaisseur de l'existence et qu'à défaut de le réconforter, elle le fasse vivre plus intensément ce que je lui raconte. Qu'il s'agisse des ruminations de mes carnets ou d'une histoire fictive, je vise la concision du vocabulaire, la netteté du trait et le rythme de la phrase, vertus flaubertiennes que Kafka a reprises à son compte. Que peu de lecteurs québécois y soient sensibles, il m’est arrivé de le déplorer, sans jamais renier ce réalisme plus évocateur que magique hors duquel il n'y a pas pour moi de vérité littéraire possible. Je ne saurais pas comment écrire autrement et je n'essaierais même pas de le faire, mon seul souci demeurant de raffiner cette prose qui serait la «signature de ma singularité», comme vous le suggérez.

VoIX ET IMAGES Un aspect commun à la fiction et aux carnets, c'est la place qu'y occupe le monde sensible. Dans le second des entretiens radiophoniques avec Stéphane Lépine, vous dites que «la sensation vraie s'entend» et survient «quand l'écriture s'efface devant son objet». Quel travail suppose la restitution de la sensation dans l'écriture? 
ANDRÉ MAJOR Ce que je vois, sens et entends, aussi loin que je remonte, j'en fais une histoire - mais pas toujours un drame. Le besoin que j'éprouve de restituer la réalité matérielle en évoquant les sensations les plus communes relève, j'imagine, de mon tempérament et du réalisme dont je parlais tout à l'heure. Pour moi, le langage doit charrier autant la matière sensible que la pensée, et c'est ainsi qu'il rend justice à la totalité du réel. Les choses ont une vie et les sensations une portée qui jouent leur rôle dans n'importe quelle histoire. Il arrive qu'un artiste - Julien Gracq, par exemple - choisisse une forme d'ascétisme pour privilégier un aspect abstrait ou quintessencié de la condition humaine. Une telle épuration ne m'intéresse pas si elle m’éloigne de cette «bienheureuse matière» dont parlait Giono. Épurer la forme pour permettre au lecteur de bien voir ce que j'évoque, voilà qui me convient parfaitement; pas question, cependant, de l'entraîner dans les coulisses de l'écriture, car ce n'est pas moi en tant que styliste qu'il doit apprécier, mais ce monde que je lui donne à voir et à sentir aussi justement que me le permettent mon esthétique et ma vision du monde. Si l'artiste ne peut sauver le monde ni fermer les yeux sur l'injustice, il peut témoigner de la beauté de cet univers périssable où il lui est donné de vivre et de mourir. Et cette beauté, oasis dans l'existence souvent désertique et tourmentée de chacun, il lui faut la restituer aussi charnellement que possible, oserais-je dire. Parmi tous ceux qui y sont parvenus, il y a le grand Tolstoï à qui rien de vivant n'a échappé. On peut lui reprocher tout ce qu'on voudra, il a tout de même recréé le monde qui était le sien avec une profondeur inégalée et un art que le temps n’a pas ridé.

voIX ET IMAGES Quel rôle jouent les lieux dans votre univers qui est toujours celui d'un marcheur?

ANDRÉ MAJOR Le lieu m'importe plus que le milieu où évoluent mes personnages, moi y compris, si tant est que je puisse me considérer comme l'un de mes personnages. Quand une situation se présente à moi, elle est située physiquement, que ce soit dans un café ou une forêt. S'il s'agit d'une histoire brève, le lieu de l'action n'est pas moins déterminant que dans un roman: c'est là que ça se passe, et il m’est impossible d'en changer en cours de rédaction, comme s'il était consubstantiel à un personnage donné. Pour "La folle d'Elvis", par exemple, inspirée par une jeune fille assise sur un banc en train de mordre dans sa pomme d'un air absent, non loin de la tour de Radio-Canada, je n'ai pas songé un instant à modifier ce décor où elle m'est apparue, par une fin d'après-midi d'été venteuse, même si une fois dans le métro mon imagination s'était mise en branle pour lui prêter le destin que l'on sait, si on a lu cette histoire. Dans un roman, le lieu doit avoir été l'objet d'une fréquentation et d'une rêverie pour m'apparaître utilisable. S'il s'agit d'un lieu familier, le souvenir me suffit; dans le cas d'un lieu étranger comme Lisbonne, il faut que je m'y sois égaré en y trouvant de quoi toucher tous mes sens. Lisbonne, je l'avais visitée en touriste il y a près de quinze ans, mais je n'en avais rapporté que quelques notes teintées de nostalgie. Passer deux mois dans un petit studio de la Graça, comme je l'ai fait en 2011, faire mes courses dans le quartier comme tout le monde, y vagabonder et $\mathrm{m}^{\prime} \mathrm{y}$ égarer, tout ça a fait germer l'idée d'un deuil au bord du Tage. Mais comme je l'ai dit, j'étais là pour réviser mes carnets, et je n'avais pu qu'imaginer une brève histoire de deuil sans deviner l'étendue qu'elle prendrait. Il y a aussi des lieux que j'invente 
de toutes pièces, si je puis dire, avec des matériaux hétéroclites que j'arrange à ma manière. Le lieu est le terreau du récit, congénitalement lié à ceux qui y circulent. Aucun personnage ne m'apparaît hors contexte, tout comme il me serait impossible de penser au Ricardo Reis de Saramago sans le voir quitter son hôtel de la Praça do Comércio, sous la pluie froide de l'automne, pour se rendre jusqu'à Santa Catarina, où le fantôme de Pessoa le rejoint.

VoIX ET IMAGES Vos personnages bâtissent, rafistolent, emménagent, s'installent - toujours un peu provisoirement - , font des plans; dans les carnets, les chalets que vous occupez successivement apparaissent. À quoi renvoie pour vous cette image de la maison?

ANDRÉ MAJOR C'est d'abord l'espace du retrait, là où, libéré de tout rôle social, on redevient plus ou moins parfaitement maître de soi: on y cuisine, on s'y délasse, on y lâche son fou, comme on dit, avant de s'y abandonner au sommeil. Le monde $s^{\prime} y$ retrouve, mis en sourdine, réduit à notre échelle, pourrait-on dire, n'interférant dans notre existence que dans la mesure où nous laissons les médias y faire intrusion. C'est là qu'on a l'impression de pouvoir être soi-même sans vergogne. Là aussi qu'on entretient des relations très intimes avec quelqu'un d'autre, qu'on accueille de vieux amis, qu'on lit, qu'on écrit, qu'on choisit la musique dont on a parfois un besoin urgent. J'ai toujours aimé apporter à cet espace vital de constantes modifications pour témoigner du temps qui passe, qu'il s'agisse de plantes, de tableaux, de meubles ou d'objets, si bien que le flux de la vie s'y reflète. Cette maison dont les murs sont les miroirs de notre personnalité, on n'envisage pas de cesser d'y vivre, tant on s'y trouve à l'aise. Et pourtant, quand on aspire à faire le vide ou à changer d'air, on la quitte volontiers pour trouver asile dans un chalet, qui ressemble souvent à un musée du patrimoine familial (vieille vaisselle, vêtements élimés mais d'autant plus confortables, meubles bancals, livres relus entre des murs dégageant une odeur de rusticité réconfortante, outils hérités du grand-père, antique poêle de fonte qui dégage une bonne chaleur les soirs de grand froid ou quand survient une panne). Autour de ce chalet, on a planté diverses espèces d'arbres, des fougères, des plantes sauvages, on a réservé un espace pour les feux de camp, beaucoup bricolé à l'intérieur comme à l'extérieur, si bien que le chalet est devenu un relais entre la maison de ville et la cabane. Car, tôt ou tard, on finit par éprouver le besoin d'une plus grande solitude, et on va dans la montagne se construire une cabane tout à fait rudimentaire avec ses quatre murs de rondins ou de vieilles planches et son toit de tôle en pente. Là, on ressent l'ivresse de s'ensauvager, ne serait-ce qu'une heure ou deux, le temps de rêvasser et de se rafraîchir si c'est au cœur de l'été ou de se réchauffer si le vent d'hiver nous a frigorifié. C'est l'abri du déserteur que l'écrivain rêve d'être parfois. Cela dit, les choses ne sont pas si simples: cette maison qui est notre véritable patrie peut devenir aussi étouffante qu'une rame de métro. Adolescent, je fuyais la maison le plus souvent possible, et c'est pour échapper à l'autorité de ma mère que j'ai fait du scoutisme. Quand je me trouvais à la campagne avec mes parents, je prenais la clé des champs, sitôt sorti de table. J'allais marcher, lire ou rêver dans les bois. Je n'ai jamais cessé de pratiquer ce vagabondage dès que j'ai un moment de liberté. Si je demeure si attaché aux lieux où je me suis installé, c'est sans doute parce que je fais constamment et en toute saison la navette entre la ville et la montagne, entre la maison et 
le chalet, entre la cuisine et l'écriture, entre la lecture et le bricolage. Il m'est impossible de m'encabaner longtemps, pas plus que de me consacrer à une seule activité, comme si je trouvais mon bonheur dans la satisfaction de tous ces besoins qu'il me semble essentiel et même urgent de satisfaire. La vieillesse finira bien par m'obliger à me bercer dans mon fauteuil en racontant n'importe quoi à n'importe qui. Un dernier rôle que je jouerai sans doute avec une certaine complaisance. Pour le moment, je trouve un grand bonheur à accompagner mes petits-fils dans la forêt où nous cueillons des chanterelles avant de faire une pause devant la cabane que la chute d'un arbre mort a fait crouler sous son poids.

VoIX ET IMAGES Par plusieurs traits, la pratique du carnet, le renoncement relatif au romanesque, le désenchantement idéologique, votre œuvre pourrait correspondre au repli souvent observé de la littérature québécoise après 1980. Mais chez vous, il n’y a pas eu de brisure, plutôt une recherche amorcée dès le début pour s'attacher à ce que vous appelez «l'élémentaire». Comment voyez-vous votre propre itinéraire au regard de cette évolution?

ANDRÉ MAJOR Très tôt, je me suis révolté contre l'espèce d'inertie spirituelle et culturelle du Québec des années 1950 et 1960 en m'engageant dans des mouvements d'action politique et en composant mes premiers écrits. Mon expérience au sein de l'équipe de la revue Parti pris a été éprouvante et décisive, comme je l'ai déjà raconté. Et, dès 1965, année de la naissance de mon fils, j'ai rompu avec toute forme de militantisme, résolu à m'engager dans la voie étroite mais exigeante de la création littéraire, tout en menant une carrière de chroniqueur littéraire, puis de réalisateur à la radio. Cette double vie, pas toujours harmonieuse, je dois le reconnaître, a duré trente-cinq ans. Au cours de cette période, il y a eu deux référendums sur l'avenir politique du Québec, et je me suis laissé enrôler pour le premier des deux. La position prise par Parizeau lors de la crise d'Oka m'a détourné définitivement du Parti québécois. Prendre parti m'est donc apparu assez tôt comme une diversion; j'ai choisi d'écrire en toute liberté, en écoutant la voix des fantômes qui hantaient mon imaginaire. Et j'ai essayé, en effet, d'aborder la réalité par le biais de l'élémentaire, guidé par l'observation et l'intuition plutôt que par une interprétation d'ordre idéologique ou moral. Jamais je n'ai été tenté de me mettre au diapason d'un courant ou d'une mode. Je voulais écrire avec mon sang, comme je l'avais déclaré à Jacques Ferron dans une de mes lettres. C'était un peu fort, je l'admets, mais cela signifiait que je ne voulais pas écrire pour écrire, mais pour voir au-delà du déjà vu et pour mieux dire comment je voyais les choses de la vie. J'ai connu, au cours de ce demi-siècle d'écriture, des périodes de stérilité que j'acceptais de traverser plutôt que de forcer ma voix quand la réalité se dérobait ou que les moyens de la saisir me manquaient. Il me fallait alors attendre que quelque chose se fasse entendre à nouveau, que des êtres et des lieux me forcent la main en quelque sorte, et alors je reprenais la plume. Il m'est arrivé de croire que j'étais peut-être un imposteur, que j'avais usurpé une fonction à laquelle je n'étais pas destiné. Un jour, j'ai fini par comprendre qu'il était normal qu'un écrivain apparaisse comme une anomalie au sein d'un milieu peu préparé à la chose, comme ce fut le cas de Kafka. Au moment où j'ai quitté la radio, je croyais que je serais un écrivain à plein temps. Eh bien, non, j'ai continué de cuisiner de plus belle, de m'occuper de mes plantes, de faire des travaux manuels, sans 
m'imposer le moindre horaire de travail tant il me semblait agréable de suivre mes impulsions. Mais j'avais tout de même mauvaise conscience, je ne sais trop pourquoi, comme si une autorité quelconque m'enjoignait d'écrire. Quand Pierre Nepveu m’a proposé de soumettre un manuscrit au jury du Prix de la revue Études françaises, je n'écrivais presque plus. Tout ce que j'avais dans mes cartons, c'était des carnets. Je m'y suis attelé et j'ai composé, parfois avec une grande joie, ce Sourire d'Anton dont des bribes avaient paru dans la revue Liberté, au début des années 1980. C'est Gilles Marcotte qui m'a suggéré le sous-titre de L'adieu au roman, qui lui semblait être le fil conducteur, plus ou moins visible, de ces notes prises entre 1973 et 1992. Pour revenir à votre question, je dirais que si je demeure fidèle à quelque chose depuis mes débuts, c'est à mon goût pour l'élémentaire, qui me ramène à l'existence saisie dans ce qu'elle a de brut et d'abrupt, là où on découvre le tourment de se savoir mortel et, avec un peu de chance, l'art de survivre à cette découverte.

VoIX ET IMAGES Vous reconnaissez-vous, et comment, dans ce que Michel Biron appelle, en se référant notamment à votre œuvre et aux déserteurs qui y sont présents, «la tentation du désert ${ }^{2} »$ ?

ANDRÉ MAJOR Je me tiens aussi loin que possible du milieu littéraire, bien que je continue de publier des livres, d'être membre d'honneur de l'UNEQ - que j'ai d'ailleurs contribué à mettre sur pied - et de jouer un rôle dans le conseil d'administration du Fonds Gabrielle Roy. Je me flatte de conserver l'amitié de quelques écrivains avec qui je peux parler de littérature ou de la vie qui passe, sans me sentir obligé de briller. Mais j'ai horreur des discours et des repas officiels, des salons du livre, de tout ce qui ressemble de près ou de loin à des mondanités. La seule perspective de parler en public me rend malade, même si j'ai longtemps fait de la radio et que je me sens à l'aise devant un auditoire d'étudiants. Je suppose que ma sauvagerie me vient des origines campagnardes de mes parents. Mais il y a aussi le fait qu'écrire a toujours été pour moi une activité quasi clandestine, si fragile et compromettante que je devais la mener à l'écart du monde extérieur. La réalité environnante n'en pénètre pas moins, bien que filtrée, dans mon espace littéraire. Je ne suis pas, comme on a pu le constater, un chantre enthousiaste de notre culture prétendument avant-gardiste et encore moins du moralisme qui nous tient lieu de religion; je me vois plutôt comme un de ces avocats du diable que certains partisans de l'unanimisme voudraient bien réduire au silence en les épinglant comme réactionnaires. Si je creuse plus profondément, je dois admettre qu'il y a chez moi une tentation du désert où il me serait loisible de me livrer à la contemplation et à la méditation, loin de la rumeur médiatique. Tentation à laquelle je résiste parce que je sais que le langage, c'est-à-dire le désir de communiquer, ne tarderait pas à me ramener au bercail. On ne peut pas vivre indéfiniment dans son propre silence, encore moins dans l'absence. Si je relis si volontiers les écrivains qui me donnent le goût d'écrire, c'est que j'ai encore besoin de m'adresser, même si c'est sous la forme du monologue, aux lecteurs qui vagabondent encore en ma compagnie. En finir avec l'écriture, j'y consentirai forcément un de ces jours, mais avec la littérature, c'est moins sûr : j'ai trop besoin d'elle. Déserter, pour moi, ce n'est

2 Michel Biron, La conscience du désert. Essais sur la littérature au Québec et ailleurs, Montréal, Boréal, coll. «Papiers collés», 2010, p. 87. 
pas m'isoler dans le désert; $c^{\prime}$ est me perdre de vue pour mieux entendre le chant du monde.

VoIX ET IMAGES Il y a dans toute votre œuvre, et depuis le début, une sorte d'humilité assumée et revendiquée, comme si vous rappeliez à l'ordre l'écrivain en relativisant son rôle et sa portée; humilité aussi bien dans les thèmes que dans la forme, ainsi que dans l'ambition de l'écriture que vous cherchez à limiter: «elle n'annonce ni les lendemains qui chantent ni l'apocalypse», dites-vous à Stéphane Lépine. Comment tenez-vous l'équilibre entre le sentiment de l'«à quoi bon?» et le désir d'écrire?

ANDRÉ MAJOR Entre le renoncement à l'écriture et le désir d'écrire, il y a une tension aussi constante et nécessaire qu'entre la ville et la montagne - je parle de la montagne plutôt que de la campagne, car pour moi la nature idéale, celle qui me fait rêver, ce n'est pas la plaine, aussi verdoyante qu'elle soit, mais la forêt ou, plus précisément, la montagne boisée - que le frère Marie-Victorin appelle les «bois montueux». La promenade n'est jamais si féconde que par les sentiers qui montent à travers l'érablière à bouleaux jaunes ou la hêtraie. Je n'imagine pas une vie où je me réveillerais avec l'obligation d'écrire, matin après matin, entre neuf heures et midi. Mais, dès le matin, quitter le chalet ou la maison, le carnet dans la poche et la canne à la main, voilà un programme selon mon cœur. Tout au long de mon vagabondage au cours duquel il $\mathrm{m}^{\prime}$ arrive de cueillir des baies ou des champignons, de repérer telle plante sauvage ou tel arbrisseau que je transplanterai en fin de journée sur notre terrain, je ruminerai sans préméditation une pensée ou un souvenir, ou bien je noterai une simple observation, assis sur le tronc d'un arbre mort. Depuis quelque temps, pour des raisons de santé, je fais trois promenades par jour, moins longues qu'auparavant, ce qui me prend passablement de temps, mais j'en tire ce qu'aucune autre activité ne pourrait m'octroyer. C'est en marchant comme je le fais, sans savoir où mes pas me mènent, que mon esprit engrange ce qu'il n'aurait jamais trouvé tout seul, dans la petite cellule où je me réfugie, une fois revenu, pour faire le tri dans cette récolte jetée en vrac sur le papier. L'«à quoi bon?», si souvent évoqué dans mes carnets, a perdu de son intensité, sans doute parce que je sais que, tant que je croirai avoir quelque chose à dire, je le dirai sans autre ambition que de le faire en beauté. Je serais heureux de savoir que mon lecteur se sent vivre plus intensément en me lisant, comme c'est mon cas en écrivant. Laisser une œuvre derrière moi, je ne m'en soucie pas le moins du monde. Cette modestie vient de la conscience que j'ai de la fragilité des choses et de l'oubli qui nous attend, moi tout autant que ceux que leur notoriété aveugle encore. Ce qu'il adviendra de la littérature telle que nous l'avons connue, bien présomptueux celui qui se risquerait à le prédire. Mais tant que j'aurai bon pied, bon œil, ma devise sera, pour paraphraser Julien Gracq, en marchant en écrivant. Mais si j'ai une phobie, c'est de perdre l'usage de mes jambes et, par conséquent, le goût d'écrire.

VoIX ET IMAGES Vous êtes un grand lecteur, vous avez réalisé des émissions littéraires pour Radio-Canada, et vous êtes aussi un lecteur fidèle qui lit toute l'œuvre des écrivains qu'il aime. Comment voyez-vous cette "communauté» des écrivains que vous lisez? Et quelle part prend-elle dans votre propre écriture? Comment redéfinit-elle selon vous l'originalité?

ANDRÉ MAJOR Les écrivains essentiels pour moi, ce sont ceux que je relis, annote et commente. C'est un aréopage assez hétéroclite de je ne sais combien d'écrivains 
parmi lesquels il y a une majorité de romanciers et quelques prosateurs, la plupart originaires d'Europe centrale et de l'Est, quelques-uns des Amériques. Ils m'ont, chacun à sa façon, ouvert des chemins et permis de prendre le large autant sur le plan spirituel que sur le plan esthétique. Mais je dois dire qu'au départ, au cours des années 1960, si j'oublie certaines influences décisives comme celles de Kafka, de Flaubert, de Rousseau, de Gombrowicz et de Céline, je me suis surtout frotté à la littérature du cru, et je me sens particulièrement redevable à Jacques Ferron et à Gabrielle Roy. J'allais oublier le frère Marie-Victorin, qui se paie le luxe d'allier l'élégance du style à la précision scientifique. Mais avec le temps, après avoir beaucoup lu pour des raisons professionnelles, j'en suis venu à élire cet aréopage d'écrivains qui demeurent depuis des années mes interlocuteurs privilégiés. Je ne sais combien de fois j'ai trouvé refuge dans les nouvelles de Tchékhov ou les récits de Tolstoï, dans les carnets de Cioran, de Canetti ou de Handke, "mon exact contemporain", comme je l'appelle. Certains écrivains que j'ai fréquentés dans ma jeunesse, même s'ils m'ont nourri et soutenu, je peine à les relire. Il y a là un deuil à faire. Je pense, entre autres, à Giono, qu'il me semble avoir aimé déraisonnablement. C'est que, tout en restant celui que j'étais, je me suis découvert d'autres exigences auxquelles répondent d'autres voix - celles de Robert Walser et du troublant et mélancolique Sebald. C'est peut-être curieux, mais j'entends la voix des écrivains quand je les lis - je les entends dans ma langue, évidemment. Cette voix touche ma sensibilité, comme le fait la musique, à cette différence que sa portée est plus étendue, plus ancrée dans le temps et dans la mémoire. Mais on trouverait peu de traces de mes lectures dans mes histoires. Sauf dans À quoi ça rime?, où Antoine évoque Pessoa et ses doubles, de même que la figure de l'oncle qu'il vient de perdre dans des termes rappelant délibérément l'éloge de l'oncle Georg que fait le narrateur d'Extinction, le dernier roman de Thomas Bernhard. Ce ne sont pas de simples clins d'œil puisqu'Antoine est un écrivain et qu'un écrivain ne cesse d'entretenir des rapports particuliers, extrêmement subtils parfois, avec d'autres écrivains. Il est vrai que c'est dans mes carnets que j'ai l'habitude de réserver mes commentaires sur mes écrivains de prédilection. À mon âge, les influences se font rares et de plus faible intensité. Ce qu'il me plaît de lire, c'est ce que j'aurais pu ou voulu écrire, en sachant que je l'aurais fait autrement, à ma manière. Là où l'influence d'un livre se manifeste, c'est quand je me promène, l'esprit léger et sans arrière-pensée, et que tout à coup les premières phrases de Voyage au bout de la nuit me reviennent: "Ça a débuté comme ça. Moi, j'avais jamais rien dit. Rien. C'est Arthur Ganate qui m'a fait parler. Arthur, un étudiant, un carabin lui aussi, un camarade ${ }^{3}$.» Ou bien, me rappelant ce personnage de Hamsun qui s'entretient avec une souris dans la hutte où il a trouvé asile, je me prends au jeu d'imaginer la conversation que je pourrais avoir avec une bestiole pareille, en supposant que je me trouve à la place de ce vagabond. Le premier écrivain qui m’a donné l'envie d'écrire, c'est Stevenson, que je n'ai cessé de relire depuis ma quatrième année d'école primaire. Après ma découverte de L'île au trésor - un prix que j'avais obtenu en fin d'année -, j'ai eu à traduire des pages de Kidnapped, que notre professeur d'anglais tenait en haute estime. Je voulais devenir David Balfour, comme j'avais

3 Louis-Ferdinand Céline, Voyage au bout de la nuit, Paris, Gallimard, coll. «Folio», 2007 [1932], p. 7. 
été Jim Hawkins. J'ai lu à peu près tout Stevenson, y compris sa correspondance. Ce virus s'est transmis à mon fils, qui est un lecteur aussi fidèle que moi du grand conteur d'Édimbourg. Et l'admiration que Borges a pour sa prose ne peut que conforter la mienne. Ce que j'ai retenu de ma longue fréquentation de Stevenson, c'est que s'il a pu explorer les ténèbres de l'âme humaine sans y sombrer, en se servant du roman d'aventures, c'est grâce à la clarté et à la fluidité de son écriture. Cela dit, il y a bien des œuvres que je n'ai jamais abordées, soit que rien en elles ne m'attire, soit que j'aie frayé dans leurs eaux sans profit aucun. J'ai plutôt tendance à revenir inlassablement aux œuvres dont les échos se répercutent toujours en moi. À bien y penser, les influences sont moins fondamentales qu'on serait porté à le croire, du moins pour l'écrivain que je suis devenu, car ce que je cherche en lisant, c'est tout autant à voir comment fonctionne l'écriture qu'à me frotter à un autre univers que le mien, à la fois étranger et assimilable au mien. Quand j'ouvre un livre, je n'ai qu'à lire une page ou deux pour savoir à quoi m'en tenir, qu'il s'agisse du style, du ton, du genre de récit qu'on me propose. Cette approche prudente - et même méfiante, je veux bien le reconnaître - ne m'empêche pas de faire des découvertes, la plus récente étant L'apocalypse des travailleurs d'un jeune écrivain portugais, Valter Hugo Mae, dont un entretien avec lui avait retenu mon attention, il y a trois ans, lors de mon séjour à Lisbonne. Quant à l'originalité, on n'a pas à la rechercher: il suffit d'écouter sa propre voix, sans essayer de se conformer à ses modèles ou de s'en démarquer à tout prix. Les influences deviennent des affluents qui alimentent le courant qui nous porte. Aussi forte qu'elle soit, une influence ne peut détourner une sensibilité de ses propres sources. Je crois d'ailleurs qu'on recherche moins ce qui nous ressemble que ce qui nous fait défaut ou ce dont on rêve.

VoIX ET IMAGES Vous n'avez jamais cessé d'exprimer une sorte d'illégitimité de votre écriture. Cette position d'autodidacte, longtemps dominante en littérature québécoise, est-elle encore la vôtre?

ANDRÉ MAJOR Quand j'ai commencé à écrire, je fréquentais des camarades en présence de qui les carences de ma formation me sautaient aux yeux, ce qui a peu à peu miné la confiance que j'avais en moi, surtout au sein de l'équipe de rédaction de Parti pris. L'expérience que j'ai dû faire à ce moment-là chez Dupuis Frères m’a à la fois libéré d'une certaine rigidité idéologique et replongé dans mon milieu d'origine. Mon manque de formation, j'ai tenté de le compenser en me livrant à une recherche qu'aucune contrainte académique n'encadrait. Même si le journalisme et la publication de mes premières œuvres m'avaient tiré de l'anonymat auquel je m'étais cru condamné, le doute me reprenait quant au bien-fondé de mes prétentions, l'exemple d'un Yves Thériault ou d'une Germaine Guèvremont ne me rassurant qu'à demi. L'époque des autodidactes était sans doute bel et bien révolue. Presque tous les écrivains de ma génération sortaient d'une faculté de lettres. Mais j'ai fini, grâce à l'amitié d'écrivains comme Ferron, Savard, Bessette et Guèvremont, par me sentir agréé à part entière. Le problème le plus tenace a été de me sentir déraciné socialement et culturellement. Il y avait un malaise entre les miens et moi, comme s'ils craignaient je ne sais quelle trahison de ma part, comme si je risquais de révéler des secrets de famille ou de les présenter sous un mauvais jour. L'écriture était un tabou à leurs yeux, comme la photographie pour certains primitifs. Raconter des histoires, c'était 
forcément révéler ce qui ne se disait pas, rouvrir de vieilles plaies. Car ces histoires, comme les journaux, ne pouvaient rien apporter de bon. Peu de temps avant sa mort, mon père m'a pour la première fois parlé de mes Histoires de déserteurs, qu'il avait relues et qu'il disait mieux comprendre maintenant. Les accepter, ça, je ne le saurai jamais. Il aura, en tout cas, été le seul membre de ma famille à avouer m'avoir lu. Tout au long de sa retraite, j'ai été son fournisseur de livres, de polars surtout, mais aussi de récits de Gabrielle Roy et d'Yves Thériault, qu'il refilait à son frère, cet oncle à qui j'ai tenté de rendre hommage dans À quoi ça rime? Pour en revenir aux conséquences qu'a pu avoir ma formation d'autodidacte, je dirais que, tout en me faisant prendre conscience de mes limites, elle m'a amené à développer une approche personnelle, assez peu méthodique, comme en témoignent les ruminations de mes carnets. Avec le temps, j'ai pactisé avec l'écrivain que je suis devenu, car comme disait ma mère, on ne peut pas donner ce qu'on n'a pas.

VoIX ET IMAGES Une manière de relativiser l'importance de la littérature tient chez vous aux autres activités auxquelles vous donnez de la place (la cuisine, le bricolage, la promenade). S'agit-il là encore de remettre la littérature à sa place?

ANDRÉ MAJOR Remettre la littérature à sa place, oui, c'est bien ce que je propose, même si cela peut paraître paradoxal dans un monde où elle végète dans la marge depuis toujours. C'est, en fait, le milieu littéraire et moi-même que je vise en relativisant l'importance de la littérature, d'abord pour éviter de vivre dans une frustration que j'ai longtemps éprouvée et ensuite parce que, force est d'en convenir, la plupart des gens préfèrent se distraire et s'amuser, une fois libérés de leurs tâches, plutôt que de s'enfermer entre les pages d'un livre, faute d'avoir été initiés à la joie que donne la lecture, faute même d'en éprouver le besoin. Je n'ai qu'à penser à mon milieu d'origine où le lecteur fait figure d'excentrique. Longtemps, en pénétrant dans une maison sans bibliothèque, j'ai eu une impression de vide. J'ai fini par m'y habituer et par considérer qu'un lecteur était un marginal heureux de l'être et que les autres, tous ces non-lecteurs, étaient bien heureux de leur côté, devant leur écran géant ou leur ordinateur. Mais je serais mal placé pour leur en faire grief, moi qui passe le plus clair de mon temps à cuisiner, à herboriser, à planter des arbres, à réparer quelque chose, quand ce n'est pas à confectionner des bâtons de marche et des cannes (j'en ai toute une collection d'essences et de formats différents, sans compter ceux que j'ai rapportés de Provence, des Alpes et de Lisbonne). Je n'ai été un homme de lettres discipliné, attelé à la tâche, de telle heure à telle heure, jour après jour, que lorsque j'entreprenais la rédaction d'une histoire que je ne pouvais interrompre sans risquer d'en perdre le fil ou de m'en désintéresser, d'où ma prédilection pour les carnets, auxquels je peux travailler une heure ou deux, quand ça me chante, puis préparer un tajine d'agneau ou des blinis farcis de saumon fumé, sortir pour flairer le vent ou, tout bonnement, vaquer aux obligations plus ou moins agréables qui sont au programme de n'importe quel retraité n'ayant aucune mission particulière à remplir. Cette existence ordinaire, si on y intègre le travail intellectuel ou le simple souci littéraire, a le mérite de me garder en contact permanent avec le courant de la vie, de ne pas me débrancher complètement du quotidien, et d'ainsi alimenter l'écriture. Tout ce qui n'est pas de la littérature finit par en devenir en y mettant son grain de sel. Comme le disait Ramuz, écrivain appartenant toujours à mon aréopage, c'est 
avec de l'antipoésie qu'on fait de la poésie. Il disait aussi que l'art était trop souvent «une greffe sur du déjà greffé» et qu'il fallait "greffer sur le sauvage». De cela je suis convaincu depuis toujours, et rien ne m'en fera démordre. C'est avec des mots qu'on écrit, bien sûr, mais encore faut-il qu'ils soient irrigués par la vie, par la conscience de la mort et par l'envie de jouir encore du temps qui nous est alloué. Cette langue «greffée sur le sauvage ", c'est l'île au trésor où j'accepterais d'être exilé s'il me fallait en venir à une telle extrémité.

VoIX ET IMAGES Vous avez campé dans vos romans une pauvreté radicale. Qu'est-ce qui vous retient dans ces personnages frustes que vous ne cherchez pas à sauver?

ANDRÉ MAJOR Cette question, je me la pose, moi aussi. Dans ma famille maternelle, dont les origines étaient à la fois écossaises et canadiennes-françaises, il n'y avait que des gens simples, pour ne pas dire frustes, la plupart analphabètes, y compris ma mère, qui a fait ses classes tardivement en compagnie de mon instituteur de père. Du côté paternel, on a été analphabète jusqu'à la génération de mon père. Lui et l'un de ses frères ont pu faire des études secondaires chez les clercs de Saint-Viateur de Rigaud, en échange de services pratiques. Deux de leurs cousins étaient médecins. Mes parents ont passé leur enfance à la campagne, où leur famille vivait dans des conditions qu'ils préféraient oublier. Quant à moi, j'ai passé les quinze premières années de ma vie dans ce qu'on appelait le Faubourg à m'lasse, non loin de l'ancien stade des Royaux. J'ai dû, pour suivre le cours classique, obtenir une bourse dite de $l^{\prime}$ Euvre des vocations, moyennant quoi je devais m'engager dans la voie sacerdotale. Chaque fois que mon directeur spirituel me demandait si j'avais enfin entendu l'appel de Dieu, je ne savais trop que répondre, me sentant coupable d'usurper la place d'un véritable élu. Ce n'est qu'après la mort de Duplessis que mon père a obtenu un salaire lui permettant de défrayer mes études. Mais un an plus tard, m'étant engagé dans une guérilla journalistique, j'ai été mis à la porte du collège et j'ai dû, après un sursis de quelques mois, trouver du travail. Nous avions alors déménagé dans le quartier Rosemont, ce que toute la famille considérait comme rien de moins qu'une promotion sociale. Nous avons toujours passé l'été à Saint-Calixte, où la famille de mon père était plus ou moins enracinée depuis pas loin d'un siècle. J'ai pu assister au lent déclin d'une économie fondée sur l'exploitation forestière et une agriculture de subsistance. Mon imaginaire s'est nourri de ce que j'ai vu et entendu là: des fermes que les jeunes désertaient et des champs laissés en friche où des chalets pousseraient comme des champignons. Toute notre tribu se retrouvait, année après année, dans des bâtiments construits, entre les deux guerres, par un Ukrainien à qui mon oncle préféré avait racheté une terre de je ne sais combien d'arpents, aux trois quarts boisée et traversée par un torrent qui se déversait dans un lac. J'ai été fasciné par un cousin de mon père qui vivait en sauvage et braconnait pour survivre. C'est lui qui m'a inspiré le personnage de Momo. Je venais d'achever ma trilogie quand j'ai vu la photo de ce cousin en première page du Journal de Montréal: un de ses neveux venait de le tuer à bout portant dans la cabane où il vivait. Fait divers peut-être, mais qui montre que je n'ai pas noirci le portrait autant qu'on a pu le croire, pas plus que je n'ai voulu l'embellir, enclin comme je l'ai toujours été à raconter les choses telles qu'elles m'apparaissaient. Quant au montagneux canton de Kilkenny et à ses habitants, je m'en suis servi assez librement pour faire vivre ce Saint-Emmanuel dont 
mon grand-père Emmanuel était la figure tutélaire. C'était un solitaire plutôt taciturne - même s'il a contracté pas moins de trois mariages - qui a scrupuleusement veillé à l'entretien de la forêt jusqu'à quatre-vingt-cinq ans, équipé d'une hache et d'une sciotte. Tant que sa jument a tenu le coup, elle a charrié les troncs d'arbres qu'il abattait. Par la suite, il débitait son bois sur place et le transportait lui-même sur un traîneau quand il y avait une bonne couche de neige. À sa mort, en 1968, j'ai hérité de sa tabatière en chêne, de sa sciotte à archet et de son chapeau de paille, que je porte toujours dès que je sors du chalet pour m'en aller promener. Ce petit monde, celui de mon faubourg montréalais tout autant que celui de la campagne, je l'ai toujours senti couler dans mes veines. Si j'avais tenté de m'en détourner, j'aurais sans doute perdu mes moyens, comme ce fut le cas de Marcel Dubé passant de son milieu d'origine à un milieu de bourgeois et de parvenus. La sensibilité et la langue prennent leur source dans l'enfance, comme tout un chacun finit par en prendre conscience, et l'artiste tout particulièrement. Ce n'est qu'avec Antoine, le protagoniste de mon premier et de mon dernier romans - de L'hiver au cœur également - , que j'ai pu aborder un territoire plus intime, dirais-je. En suivant les traces d'Antoine, mon alter ego, je n'ai fait que poursuivre ailleurs mon travail de romancier parce que le monde de mon enfance et tout ce qu'il générait en moi était épuisé. En 2001, j'ai quitté pour de bon le paysage qui avait été si inspirant pour moi depuis ma prime enfance jusqu'au milieu des années 1990. Mais il n'y a pas eu de véritable rupture, si on considère qu'Antoine appartient au même monde que moi et qu'il n'a pas cherché refuge, lui non plus, dans une autre société, même pas celle à laquelle son statut d'écrivain aurait dû l'agréger, tout simplement parce qu'il demeure un irréductible solitaire. Cela dit, si la littérature n'avait pas tant compté pour moi et si j'avais été de la même trempe que mes frères, je serais probablement devenu quelqu'un d'autre, j'aurais vécu une autre vie, que j'imagine mal; j'aurais peut-être même été l'un des déserteurs de mes histoires.

VoIX ET IMAGES Vous êtes aussi un épistolier assidu. Vos correspondances avec Jacques Ferron et avec Félix-Antoine Savard ont été publiées. Quelle place occupe aujourd'hui la correspondance?

ANDRÉ MAJOR On peut dire que j'aurai été un épistolier assez assidu entre les années 1960 et la fin du siècle, soit une quarantaine d'années. Avec certains correspondants comme Pierre Vadeboncœur, les échanges ont duré plus de trente ans, presque toujours dictés par la parution de nos ouvrages respectifs. C'est la littérature qui en était le thème dominant, souvent associée aux rapports qu'elle entretenait avec notre vie. Cette correspondance compte une petite centaine de lettres. J'ai moins correspondu avec des amis de mon âge pour la bonne raison que nous nous rencontrions régulièrement. Quand l'un de nous était à l'étranger, les échanges devenaient plus fréquents et nourris. Ces échanges avaient le mérite de faire le point sur ce que chacun devenait et de mesurer ce qui nous rapprochait ou nous distinguait, mais que ces échanges aient eu des retombées sur mon travail proprement littéraire, je ne me risquerais pas à l'affirmer, même après avoir relu les deux correspondances publiées jusqu'à maintenant et, plus récemment, celle que j'ai entretenue avec Vadeboncœur de 1972 à 2005. Ce que j'ai constaté, c'est qu'en dépit de tout ce qui nous séparait - aussi bien l'âge que la vision du monde -, il y avait entre nous une véritable fraternité et 
une franchise assez stimulante. Cette relecture m'a fait remonter le cours du temps, et j'ai vu à quel point tout cela appartient à une époque et à un esprit révolus. De tels échanges seraient improbables aujourd'hui, ne serait-ce qu'à cause de ce mode de communication express qu'est le courriel. On ne s'écrit plus pour aborder un thème ou une question délicate, mais pour échanger des informations, des sites à consulter ou pour fixer un rendez-vous à l'occasion duquel on espère pouvoir développer ce qui ne peut, en fait, se développer qu'au long d'une bonne vieille lettre manuscrite, suivie d'un ou de deux post-scriptum. Cela dit, le lecteur que je suis plonge avec délectation dans la correspondance d'un Tchékhov, comme je l'ai dit, ou bien dans celle d'un Kafka, qu'il écrive à ses amis ou, mieux encore, à ses fiancées Felice et Milena. On y découvre les raisons du profond désarroi dont témoigne si fortement et si singulièrement son œuvre romanesque inachevée et qui était probablement inachevable - un désarroi que chacun a éprouvé, peu ou prou, devant l'absurdité du monde et le fait de vivre en se sachant mortel. Je me souviens qu'enfant, il m'arrivait d'être pris de vertige au bord du gouffre qui s'ouvrait devant moi, tout simplement parce que j'ignorais pourquoi j'étais au monde et comment je devais vivre. Ce savoirvivre, qui n'est pas toujours un gai savoir, c'est mon insatiable curiosité qui a fini par $\mathrm{m}^{\prime}$ en inculquer les rudiments en puisant dans l'art et dans le monde naturel - bien plus que dans la lamentable histoire humaine, intarissable source de mélancolie pour tout observateur le moindrement sensible. C'est bien pourquoi la beauté occupe tant de place dans ma vie - cette beauté qui passe pour moi par le langage, par le souci de coller à l'humble vérité qu'on arrache au désordre d'un monde apparemment sans queue ni tête. Dans le lieu commun du langage, je crois qu'on parvient, sinon à surmonter son désarroi, du moins à le partager avec ses semblables. Si c'est là une illusion, c'est bien la seule que je n'ai pas encore perdue. 